\title{
TI.135.1
}

\section{eduroam US Advisory Committee Charter}

- PDF: eduroam-US Advisory Committee Charter v1.0.pdf

- Text: eduroam-US Advisory Committee Charter v1.0.txt

\section{More Information}

\begin{tabular}{|l|l|}
\hline Repository ID & TI.135.1 \\
\hline Persistent URL & http://doi.org/10.26869/TI.135.1 \\
\hline Title & eduroam US Advisory Committee Charter \\
\hline Authors & Mike Zawacki, Nick Roy, Rob Carter \\
\hline Sponsor & Kevin Morooney \\
\hline Review & \\
\hline Status & Preserve \\
\hline Publish Date & $09-24-2019$ \\
\hline DOI & $10.26869 / T I .135 .1$ \\
\hline Signature & \\
\hline Deprecated & No \\
\hline Future Review & $08-08-2021$ \\
\hline Supersedes & \\
\hline Format & PDF, text \\
\hline Related Docs & \\
\hline Development Location & https://docs.google.com/document/d/1PDOnbFVq_BGrt-fK8_q7_J07BPuthhwuuHPdWP3zRno \\
\hline IP Framework & CC BY 4.0 \\
\hline Subject Tags & charter, eduroam, service \\
\hline Notes & version 1.0 \\
\hline
\end{tabular}

